\title{
Characterization of Optically-Reconfigurable Metasurfaces by a Free Space Microwave Bistatic Technique
}

\author{
Houssemeddine Krraoui ${ }^{1, *} \mathbb{1}$, Charlotte Tripon-Canseliet ${ }^{1}$, Ivan Maksimovic ${ }^{1}$, Stefan Varault ${ }^{2}$, \\ Gregoire Pillet ${ }^{2}$, Stefano Maci $^{3}$ and Jean Chazelas ${ }^{2}$ \\ 1 Physics and Material Science Laboratory, Sorbonne Université, CNRS-ESPCI, 75005 Paris, France; \\ charlotte.tripon-canseliet@espci.fr (C.T.-C.); ivan.maksimovic@espci.fr (I.M.) \\ 2 Thales Defense Mission Systems, 78990 Élancourt, France; stefan.varault@fr.thalesgroup.com (S.V.); \\ gregoire.pillet@thalesgroup.com (G.P.); jean.chazelas@fr.thalesgroup.com (J.C.) \\ 3 Department of Information Engineering and Mathematics, University of Siena, 53100 Siena, Italy; \\ macis@dii.unisi.it \\ * Correspondence: houssemeddine.krraoui@espci.fr; Tel.: +33-063-514-7982
}

Received: 13 May 2020; Accepted: 22 June 2020; Published: 25 June 2020

\begin{abstract}
Microwave performance extraction of optically-controlled squared frequency-selective surface (FSS) structures printed on highly resistive (HR) silicon substrate are presented, from a innovative bistatic microwave photonic characterization technique operating in the 40 to $60 \mathrm{GHz}$ frequency range, commonly used for radar cross section (RCS) measurements. According to typical physical photon absorption phenomenon occurring in photoconductive materials, these structures demonstrate experimentally a bandpass filtering frequency response cancellation through reflection coefficient measurements, under specific incident collective illumination in the Near-infrared region (NIR). This behaviour is attributed to their microwave surface impedance modification accordingly to the incident optical power, allowing ultrafast reconfigurability of such devices by optics
\end{abstract}

Keywords: metasurfaces; photoconductivity; ultrafast reconfigurability; radars systems

\section{Introduction}

Metasurfaces (MTS) can act today as a new generation of frequency-selective surface (FSS) which have significantly contributed to advanced modern communication systems enhancements. Applications of MTSs at microwaves encompass those of FSS, namely, phase shifting elements for reflector antennas [1-6], filtering interfaces in radomes to reduce RCS levels in military platforms [7], as well as new generation of antennas [8-10]. Today, a complementary frequency and phase agility in MTS design is expected in next future industrial systems, as in 5G communications where a dynamic frequency allocation at end user level is required. In this area, main research works have been largely devoted to electronic solutions suggestion, such as by local electrical switching [11] from planar active devices (diodes) networks, or by substrate material permittivity change under specific electrical bias, using phase change materials.

Optical control becomes a disruptive alternative providing many advantages such as ultrafast activation speed, and EMI-protected external control. In this context, for the first time, this paper describes the development of a novel accurate free space experimental set-up dedicated to microwave reflection/transmission Fresnel coefficients measurements. This environment also incorporates optical illumination system for frequency response change demonstration of microwave FSS structures under light excitation. 
Our approach was born from research works on exploitation and exaltation of photoconductive properties of semiconducting materials, in microwave switching devices when submitted to photons flux bringing an energy by absorption superior to their band gap energy $E_{g}$.

In such materials, under light excitation, at first order of approximation, photogenerated free carriers either electrons or holes in the illuminated area change locally material conductivity by a value of $\Delta \sigma$. As material absorption coefficient determining light penetration depth value $\alpha$ in the material is dependent to the wavelength $\lambda$ of the incident optical signal, coupled parameters such as optical wavelength and material thickness must be carefully chosen for photoconductive effect existence [12-16]. In our case, NIR light appears as a suitable wavelength illumination for light/matter interaction exaltation in silicon material, which also acts as standard substrate material for integrated electronic devices manufacturing.

This publication presents experimental microwave response results of optically-controlled FSS structures Figure 1 assumed by planar capacitive square metallic patterns supported by a HR silicon substrate whose relative complex permittivity expression following Drude model (1) is a function of the angular frequency $\omega, \sigma$ is the substrate conductivity , $\varepsilon_{\text {inf }}$ being the high frequency substrate permittivity, and $\epsilon_{0}$ the vacuum permittivity.

$$
\varepsilon_{r}=\varepsilon_{\mathrm{inf}}+i \frac{\sigma_{0}+\Delta \sigma}{\omega \epsilon_{0}}
$$

Through light/matter interaction, the photon energy absorption produces a material conductance change $\Delta \sigma(2)$, which is strongly dependent on carriers dynamics such as lifetime $\tau$ and mobility $\mu_{n, p}$, in association with the incident photon flux $\Phi(t)$, quantum efficiency $\eta$ and effective volume of the light/matter interaction area $w A$ [17].

$$
\Delta \sigma=q \frac{\eta \tau\left(\mu_{n}+\mu_{p}\right) \Phi}{w A}
$$

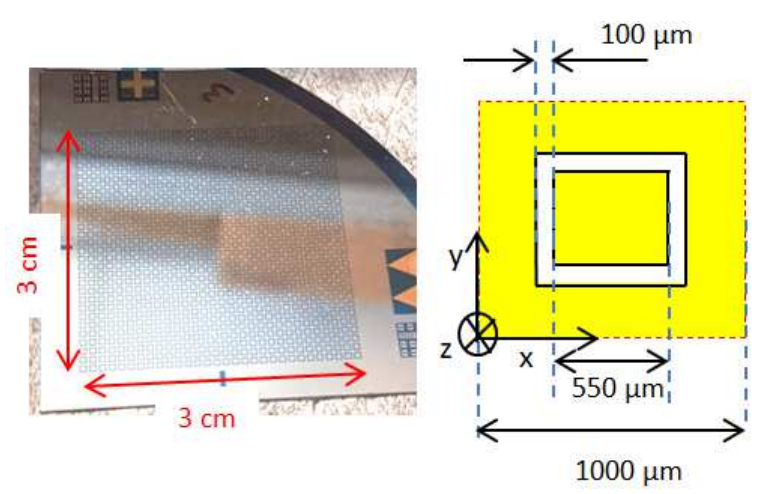

Figure 1. Optical image of frequency-selective surface (FSS) structures on Si substrate and unit cell physical description.

\section{Characterization Technique}

Compared to other competitive material characterisation techniques operating in guided environment, free space method fits for broadband material electromagnetic properties qualification and simultaneous optical illumination implementation needs. Furthermore, thanks to a homemade three-step calibration and a genetic algorithm-based data postprocessing technique, frequency-dependent material permittivity, permeability and conductivity parameters are obtained simultaneously with accuracy $[18,19]$. Schematic of the free space bistatic measurement system given in Figure 2, which consists of a pair of microwave horn antennas connected to a vectorial network analyser (VNA), acting as microwave signal transmitter and receiver. 


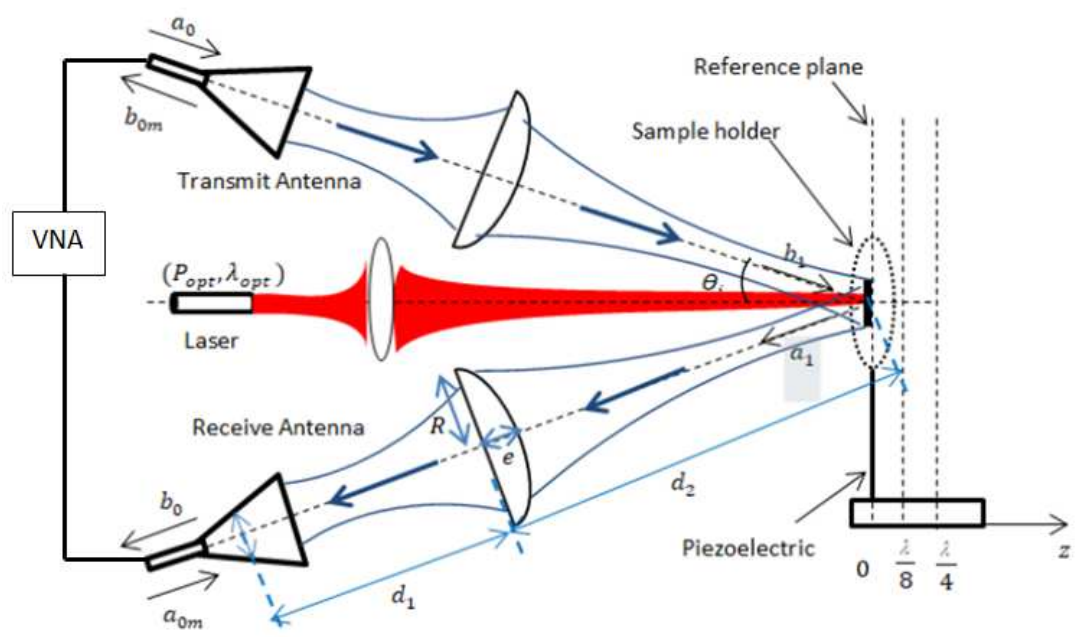

Figure 2. Schematic description of the experimental set-up.

Microwave Teflon lenses placed between antennas and the material under test (MUT), under a small signal incidence angle of $28^{\circ}$ with the respect to the normal of the MUT, are added for the guarantee of microwave signal focus in a foot print at order of MUT surface dimensions and microwave signal wavelength value. These components are mandatory for occurring diffraction effects minimization at the MUT edges responsible of measurements errors.

The focusing system has been designed for a nominal microwave frequency of $50 \mathrm{GHz}$. In this configuration, the microwave lenses must be localized at a distance $d_{1}$ equal to $31.85 \mathrm{~cm}$ from transmitter or receiver delivering a microwave signal waist of $w_{1}$ is equal to $1.24 \mathrm{~cm}$, and at a distance $d_{2}$ to the MUT equal to $18.15 \mathrm{~cm}$.

The lowest possible angle $\left(\theta_{i}=28^{\circ}\right)$ is determined by the size of transmitter and receiver. This limitation is then governed by the MUT dimensions $w$, the distance between antennas and MUT $d_{1}+d_{2}$, as well as $3-\mathrm{dB}$ angular beamwidths of each antenna in E-plane and H-plane [20]. In our configuration, the highest angle of $33^{\circ}$ has been calculated for a $1 \mathrm{~cm}^{2}$ of MUT surface and $50 \mathrm{~cm}$ of distance with $20^{\circ}$ of antennas angular beamwidths. S-parameters measurements are performed at the distance ( $D=d_{1}+d_{2}$ ) above the far field region limit corresponding to $50 \mathrm{~cm}$ at the setup lowest frequency of $40 \mathrm{GHz}$.

An optical system consisting of a LUMICS thermally-regulated cw laser diode (model:LU0808/0980/1064D100/100/100-U30AA) and a set of optical lenses is inserted between microwave horn antennas in order to realize a collimated illumination of $2.5 \mathrm{~cm}^{2}$ at low incident angle on the top face of the sample in order to avoid optical back reflection signal on the laser source.

\section{Calibration Technique}

After a standard SOLT calibration procedure executed in the reference plane of the coaxial port inputs of each horn antenna, an additional homemade calibration stepis performed for measurements deembedding at the front face of the sample. This step is based on scattering parameters measurement between the two horn antennas connected to the VNA, obtained from a metallic plate used as reference short circuit and placed at three different phase references (SSS) thanks to a piezoelectric sample holder, solving errors measurements made in a quadrupole configuration as in Figure 3. These error factors are attributed to multiple reflections between transmitting and receiving signals through MUT top face, as well as from non protected EM environment. As in Figure 2, $a_{0}$ and $b_{0}$ coefficients correspond to incident and reflected voltage wave amplitudes from transmit and receive antennas, respectively, and $b_{1}$ and $a_{1}$ coefficients being incident and reflected voltage wave amplitudes at a reference plane located at the top face of the MUT, respectively, as indicated in the schematic given in Figure 3. 


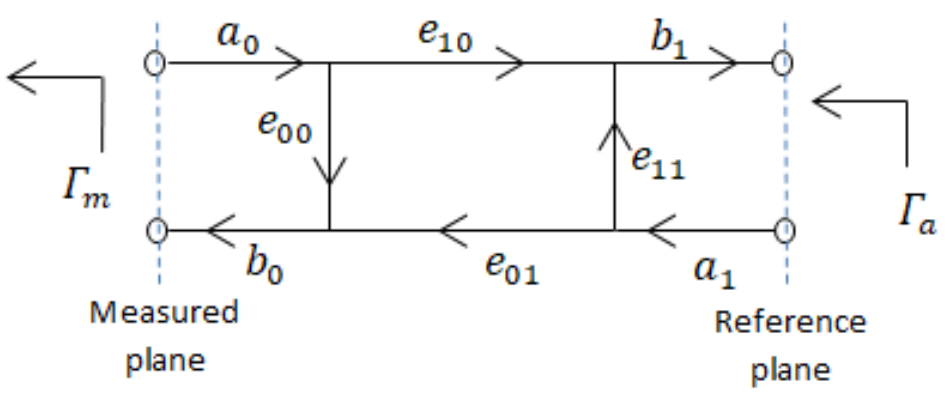

Figure 3. Quadrupole representation of microwave set-up errors.

This latter helps in the definition of the relationship between effective reflection coefficient of the MUT $\Gamma_{a}$ and raw reflection coefficient $\Gamma_{m}$ from $S_{21}$ coefficient measurement, through error coefficients introduction $\left(e_{00}, e_{01}, e_{10}\right.$ and $\left.e_{11}\right)$.

From equivalent circuit in Figure 3 , we can write that $b_{0}$ coefficient is equal to $e_{00} a_{0}+e_{01} a_{1}, b_{1}$ is equivalent to $e_{10} a_{0}+e_{11} a_{1}$ and that $\Gamma_{m, a}$ are defined by $b_{0,1} / a_{0,1}$, respectively. Then, the corrected and raw reflection coefficients $\Gamma_{a}$ and $\Gamma_{m}$, respectively, are expressed as in (3),

$$
\Gamma_{m}=\frac{a \Gamma_{a}+b}{c \Gamma_{a}+1}
$$

where $a=\left(e_{10} e_{01}-e_{00} e_{11}\right), b=e_{00}$ and $c=-e_{11}$.

To estimate the corrected reflection coefficient, it is necessary to determine the unknown parameters $a, b$ and $c$, as it is reachable by testing three calibration standards at different phase reference planes delivering known $\Gamma_{a}$.

The first calibration standard consists of a metallic sample acting as a short circuit, placed at the reference plane. The second and third calibration standards are defined by moving this metallic sample by a distance of $\lambda_{z m} / 8$ and $\lambda_{z m} / 4$, respectively, from the reference plane, leading to their reflection coefficient expression derived from the standard transmission line theory (4):

$$
\Gamma_{a, i}=\frac{\left(j \tan \left(\beta_{z m} z\right)-1\right)}{\left(j \tan \left(\beta_{z m} z\right)+1\right)}
$$

With, for $\mathrm{i}=2, z=\lambda_{z m} / 8$ and $\mathrm{i}=3, z=\lambda_{z m} / 4$. The Equation (3) can be rewritten in analytic form as in (5) leading to a better definition of error coefficients $a, b$ and $c$.

$$
\Gamma_{a, i} a+b-\Gamma_{a, i} \Gamma_{m, i} c=\Gamma_{m, i} \quad\{i=1,2,3\}
$$

Experimental reflection coefficient magnitude and phase values of $\Gamma$ of a $2 \mathrm{~cm}^{2}$ metallic sample, placed at the reference plane, are provided in Figure 4 in frequency, attesting calibration technique accuracy. 

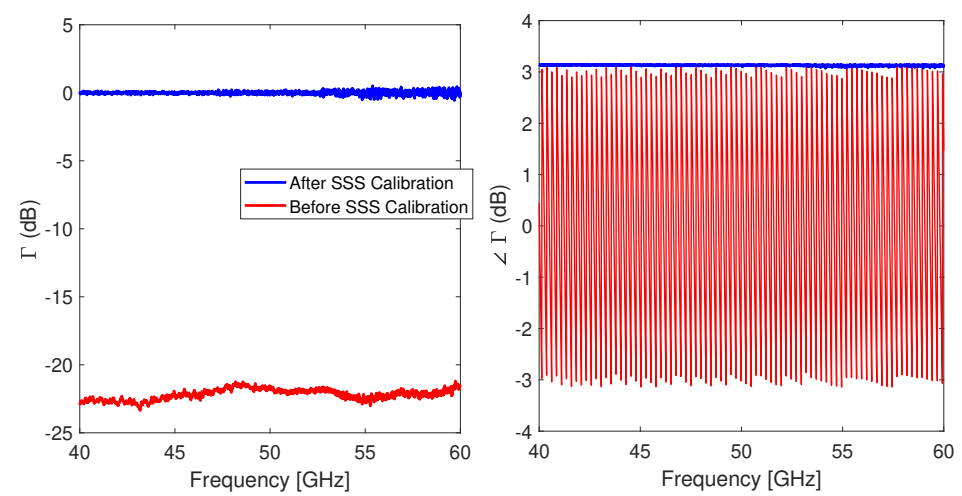

Figure 4. Experimental reflection coefficient values in magnitude and phase ofa $2 \mathrm{~cm}^{2}$ metallic plate as a function of microwave frequency before and after SSS calibration $\left(\theta_{0}=28^{\circ}\right.$ and $\left.\mathrm{D}=50 \mathrm{~cm}\right)$

\section{Microwave Characterization of HR Silicon Material under NIR Illumination}

Using the same experimental set-up, S-parameters measurements of a square HR-silicon sample of with a $2 \mathrm{~cm}^{2}$ cross section and $500 \mu \mathrm{m}$ thickness are performed at room temperature under optical power up to $10 \mathrm{~W}$ and wavelength of $805 \mathrm{~nm}$.
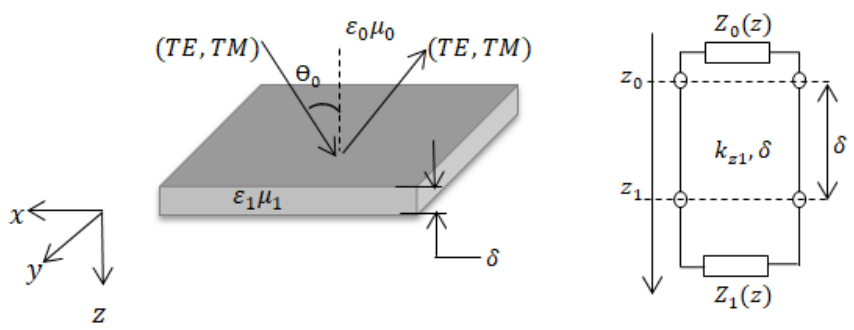

Figure 5. Two independent reflection measurements.

Under light illumination, as described in Figure 5, microwave incident plane wave is polarised either perpendicularly or parallel to its plane of incidence (TE or TM mode respectively) onto successively air/plasma, plasma/HR-Si and $\mathrm{HR} \mathrm{Si}$ /air layers interfaces, synthesized as shunt tranverse impedances representation for each individual propagating medium.

$$
Z_{2}\left(z_{1}\right)=Z_{0}
$$

Boundary conditions on tangential fields can be applied at $z=z_{0}$. Conservation of both tangential/normal electric and normal/tangential magnetic fields through each interface may be achieved by enforcing continuity of transverse impedance $Z_{1}^{(T M)}\left(z_{0}\right)=Z_{2}^{T M}\left(z_{0}\right)$ and $Z_{1}^{(T E)}\left(z_{0}\right)=$ $Z_{2}^{T E}\left(z_{0}\right)$.

Then,

$$
Z_{1}^{(\alpha)}\left(z_{0}\right)=\chi^{(\alpha)} \frac{Z_{0}^{(\alpha)}+\chi^{(\alpha)} \tanh \left(j k_{z 1} \delta\right)}{\chi^{(\alpha)}+Z_{0}^{(\alpha)} \tanh \left(j k_{z 1} \delta\right)}, \quad\{\alpha=T M, T E\}
$$

From (7), theoretical respective reflection coefficients $\Gamma_{0}^{(T M)}\left(\varepsilon_{r}^{*}, \mu_{r}^{*}\right)$ and $\Gamma_{0}^{(T E)}\left(\varepsilon_{r}^{*}, \mu_{r}^{*}\right)$ can be established and expressed as follows,

$$
\Gamma_{0}^{(\alpha)}\left(\varepsilon_{r}^{*}, \mu_{r}^{*}\right)=\frac{Z_{1}^{(\alpha)}\left(z_{0}\right)-Z_{0}^{(\alpha)}}{Z_{1}^{(\alpha)}\left(z_{0}\right)+Z_{0}^{(\alpha)}}, \quad\{\alpha=T M, T E\}
$$


The free space impedance and plasma characteristic impedance, $\left(Z_{0}^{\alpha}, \chi^{\alpha}\right)$, for the TE and TM modes are given for different angles of incidence $\theta_{0}$ as

$$
\begin{array}{r}
Z_{0}^{(T M)}=\eta_{0} \cos \theta_{0} \\
Z_{0}^{(T E)}=\frac{\eta_{0}}{\cos \theta_{0}} \\
\chi^{(T M)}=\frac{k_{z 1}}{\omega \epsilon_{r 1}^{*} \epsilon_{0}} \\
\chi^{(T E)}=\frac{\omega \mu_{r 1}^{*} \mu_{0}}{k_{z 1}}
\end{array}
$$

with $k_{z 1}=\sqrt{k^{2}-k_{0}^{2} \sin ^{2} \theta_{0}}$ is the propagation constant along the normal direction to the dielectric.

Where $k=\omega \sqrt{\epsilon_{1} \mu_{1}}$ and $\eta_{0}=\sqrt{\frac{\mu_{0}}{\epsilon_{0}}}$ is the free space impedance.

From experimental results, photoconductive material complex permittivity is extracted by using a numerical optimization procedure based on genetic algorithm [18].
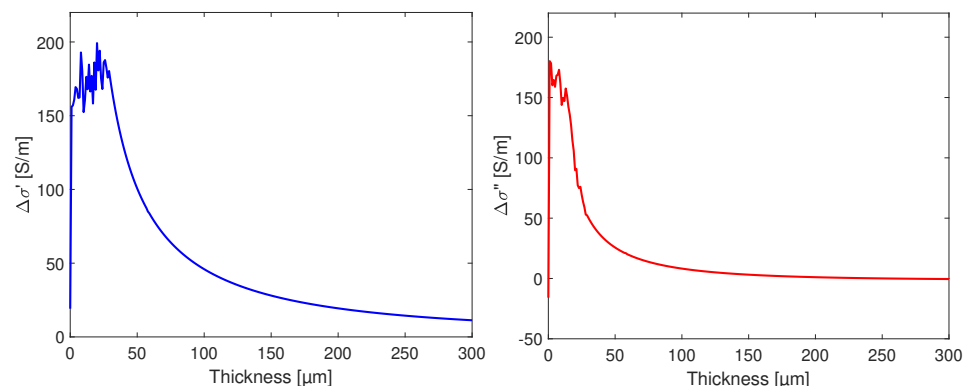

Figure 6. Extraction of the HR Si substrate photoconductance $\Delta \sigma$ (left) Real part and (right) imaginary part according to the plasma depth $t_{p}$ of the HR Silicon illuminated by optical power Popt $=6 \mathrm{~W}$ at wavelength $\lambda=805 \mathrm{~nm}$ and $\mathrm{F}=50 \mathrm{GHz}$.

From (2), photoconductance value $\Delta \sigma$, attributed to the electron/hole pairs plasma layer presence induced by light absorption inside the semiconducting material, is deduced as a function of microwave frequency. In complement, from shunt impedance de-embedding calculations, a plasma profile $\Delta \sigma$ is obtained as a function of depth inside the material, as in Figure 6 at a fixed microwave frequency of $50 \mathrm{GHz}$, and Figure 7 shows the plasma conductivity according the optical power of the NIR source and a fixed plasma depth $t_{p}=50 \mu \mathrm{m}$. From fitting calculations, material absorption coefficient $\alpha=234 \times 10^{3} \mathrm{~m}^{-1}$, and carrier lifetime of $\tau=158 \times 10^{-9} \mathrm{~s}$ at $805 \mathrm{~nm}$ are simultaneously extracted from measurement results.
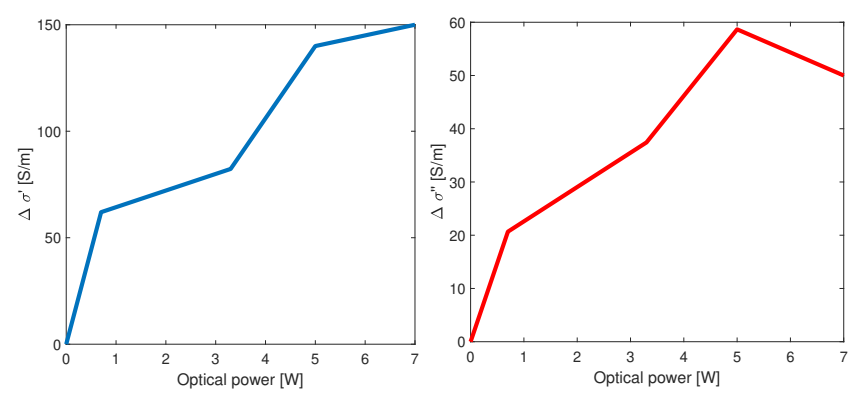

Figure 7. Extraction of the HR Si substrate photoconductance $\Delta \sigma$ in (left) Real part and (right) imaginary part according to incident optical power $P_{o p}$ at optical wavelength $\lambda=805 \mathrm{~nm}$ and microwave signal frequency $\mathrm{F}=50 \mathrm{GHz}$. 
Exponential photoconductance decrease from the surface of sample is experimentally observed, on both real and imaginary parts, in very good agreement with theoretical predictions considering light penetration length at a given optical wavelength in the absorption band of the photoconductive material [21].

\section{Design and Characterization of Optically-Controlled Microwave FSS}

Coplanar slot based MTS structures have been designed by $550 \times 550 \mu \mathrm{m}^{2}$ square metallic patches with a $100 \mu \mathrm{m}$ gap with surrounding planar ground plane in Figure 1. Metallic parts are fabricated by optical lithography of a $2 \mu \mathrm{m}$-thick layer of gold deposited on a $250 \mu \mathrm{m}$ thick HR Si substrate after a SiN dielectric layer deposition and etching at edges of conductive FSS parts.

The beam spot cross section of collective optical illumination of $2.5 \mathrm{~cm}^{2}$ at a wavelength of $805 \mathrm{~nm}$ and a maximum incident power of $10 \mathrm{~W}$ is applied at the top face of the MUT. In dark environment, MTS-based MUT is expected to demonstrate a microwave bandpass filtering behaviour centred at a frequency of $46 \mathrm{GHz}$. Reflection coefficient simulations have been achieved by electromagnetic (EM) full-wave analysis (Ansys-HFSS software), as shown in Figure 8 where conduction losses of the HR-Silicon substrate play a significant role in the frequency response.

Under optical illumination, magnitude and phase values of $\Gamma$ obtained from $S_{21}$ measurements reveal impedance mismatching response of MTS structures operated by light absorption through photoconductive effect operation occurring in the substrate opened ring area formed by the gap between the metallic patches and surrounding ground plane.
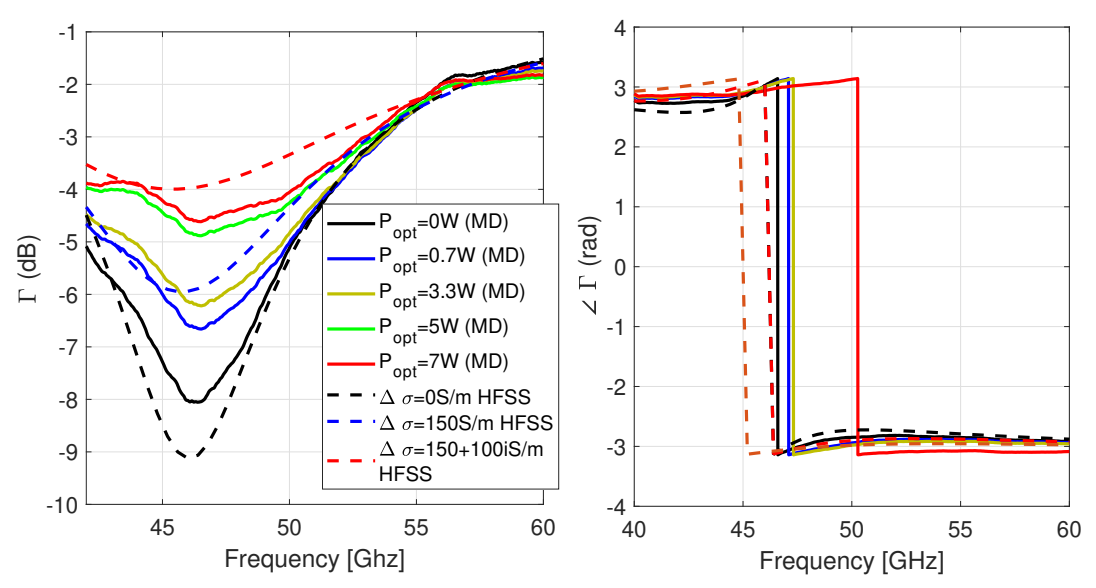

Figure 8. Measured (MD) and Simulated (SD) magnitude $\Gamma(d B)$ and phase $\angle \Gamma(\mathrm{rad})$ values of reflection coefficient under different optical illumination power from FSS structures on HR Silicon substrate with a thickness of $250 \mu \mathrm{m}$.

As reported in Figure 8, dual magnitude decrease and null-phase shifting of the reflection coefficient $\Gamma$ indicate the progressive device microwave response transition from a FSS to short-circuit type in the optical power range of 0 to $7 \mathrm{~W}$ at an optical wavelength of $\lambda=805 \mathrm{~nm}$, directly attributed to a surface impedance $Z_{s}$ modification of FSS structure by optical control. Experimental results have been then validated by a complementary full-wave analysis (Ansys-HFSS software) integrating the photogenerated plasma ring layer with experimental complex data obtained in Figure 1. Free carriers presence during light excitation affect the magnitude of $\Gamma$ through its real part, and the resonance frequency shift from its imaginary part as shown in Figure 8. Resistive losses in silicon are responsible of the detected frequency notch as we consider a perfect dielectric substrate for dark environment representation in EM simulations.

FSS surface impedance $Z_{s}$ is then directly extracted from input impedance $Z_{\text {in }}=Z_{D} \cdot Z_{s} /\left(Z_{D}+Z_{s}\right)$ with $Z_{D}$ modelling the impedance of the thin $\mathrm{SiO} 2$ dielectric layer $(2 \mu \mathrm{m})$ covering the HR-Silicon substrate $\left(\varepsilon_{r}=11.9\right.$ and thickness of $\left.250 \mu \mathrm{m}\right)$ inducing a resonant frequency shift between $\Gamma$ and $Z_{s}$. 

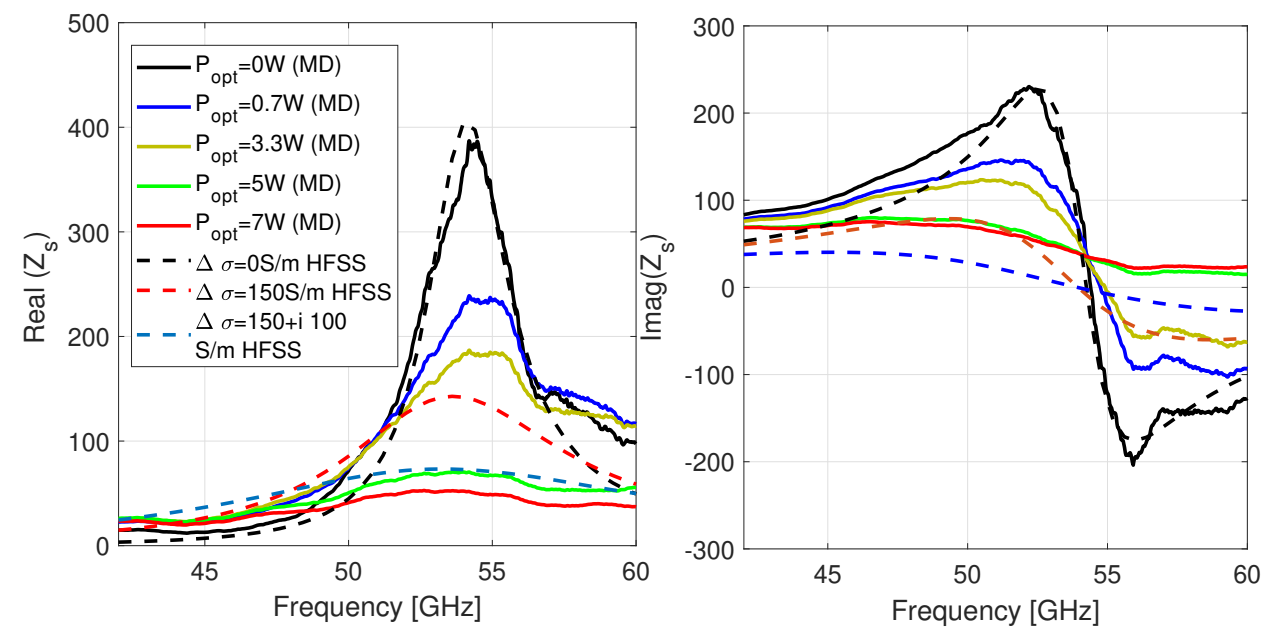

Figure 9. Measured data (MD) and Simulated with HFSS (left) Real $\left(Z_{S}\right)$ and (right) Image $\left(Z_{s}\right)$ under different optical illumination $(\Delta \sigma *)$ of MTS printed on HR Silicon substrate of thickness $250 \mu \mathrm{m}$.

As reported in Figure 9, when increasing optical power, plasma conductivity enhancement $\Delta \sigma$ tends to shunt FSS structures, where a decrease of the real part of $Z_{s}$ as well as a low slope of the imaginary part of the surface impedance $Z_{s}$ respectively is measured. Identical frequency-dependent behaviour of $Z_{s}$ is observed through EM simulations by changing plasma conductivity value $\Delta \sigma$ in the gap region.

\section{Conclusions}

A thin material layer characterization technique based on free space bistatic radar cross section measurements has been developed in order to extract the microwave complex conductivity and photoconductivity of a HR Silicon substrate. From this new microwave photonic set-up associated to a complementary homemade calibration technique, material absorption coefficient $\alpha$, as well as carrier lifetime $\tau$ are experimentally determined. By suing this set-up, surface impedance extinction of microwave FSS structures designed, fabricated and tested on HR Si wafer, induced by a collective IR illumination have been experimentally demonstrated for the first time, in the 40-60 GHz frequency range.

These results open news routes for next generation of radar and satcom systems where ultrafast frequency agility could be operated by external optical control.

Author Contributions: Conceptualization, C.T.-C., G.P, J.C; methodology, C.T.-C., G.P., S.M., J.C.; investigation, C.T.-C., G.P., S.V.; data curation, H.K ; writing, C.T.-C., H.K.; Software, I.M. project administration, J.C.; funding acquisition, J.C. All authors have read and agreed to the published version of the manuscript.

Funding: This research was funded by THALES Defence Mission Systems, Elancourt, France.

Acknowledgments: This work has been supported by THALES Defence Mission Systems, Elancourt, France.

Conflicts of Interest: Conflicts of Interest: The authors declare no conflicts of interest. The funders had no role in the design of the study; in the collection, analyses, or interpretation of data; in the writing of the manuscript, or in the decision to publish the results.

\section{References}

1. Roy Chowdhury, D.; Singh, R.; O’Hara, J.F.; Chen, H.T.; Taylor, A.J.; Azad, A.K. Dynamically Reconfigurable Terahertz Metamaterial through Photo-Doped Semiconductor. Appl. Phys. Lett. 2011, 99, 231101. [CrossRef]

2. Berry, C.W.; Wang, N.; Hashemi, M.R.; Unlu, M.; Jarrahi, M. Significant Performance Enhancement in Photoconductive Terahertz Optoelectronics by Incorporating Plasmonic Contact Electrodes. Nat. Commun. 2013, 4, 1-10. [CrossRef] [PubMed] 
3. Manasson, V.A.; Sadovnik, L.S.; Moussessian, A.; Rutledge, D.B. Millimeter-Wave Diffraction by a Photo-Induced Plasma Grating. IEEE Trans. Microw. Theory Tech. 1995, 43, 2288-2290. [CrossRef]

4. Vardaxoglou, J.C. Optical switching of frequency selective surface bandpass response. Electron. Lett. 1996, 32, 2345-2346. [CrossRef]

5. Manasson, V.A. An Optically Controlled MMW Beam-Steering Antenna Based on a Novel Architecture. IEEE Trans. Microw. Theory Tech. 1997, 45, 1497-1500. [CrossRef]

6. Brand, G.F. Remote Millimeter-Wave Beam Control by the Illumination of a Semiconductor. IEEE Trans. Microw. Theory Tech. 2000, 48, 855-857. [CrossRef]

7. Li, H.; Wang, B.Z.; Zheng, G.; Shao, W.; Guo, L. A Reflectarray Antenna Backed on FSS for Low RCS and High Radiation Performances. Prog. Electromagn. Res. C 2010, 15, 145-155. [CrossRef]

8. Faenzi, M.; Minatti, G.; Gonzalez-Ovejero, D.; Caminita, F.; Martini, E.; Della Giovampaola, C.; Maci, S. Metasurface Antennas: New Models, Applications and Realizations. Sci. Rep. 2019, 9. [CrossRef] [PubMed]

9. Alibakhshikenari, M.; Virdee, B.S.; See, C.H.; Abd-Alhameed, R.; Falcone, F.; Limiti, E. A Novel 0.3-0.31 $\mathrm{THz}$ GaAs-Based Transceiver with on-Chip Slotted Metamaterial Antenna Based on SIW Technology. In Proceedings of the 2019 IEEE Asia-Pacific Microwave Conference (APMC), Singapore, 10-13 December 2019; pp. 69-71. [CrossRef]

10. Alibakhshikenari, M.; Virdee, B.S.; Shukla, P.; See, C.H.; Abd-Alhameed, R.; Falcone, F.; Limiti, E. Meta-Surface Wall Suppression of Mutual Coupling between Microstrip Patch Antenna Arrays for THz-Band Applications. Prog. Electromagn. Res. Lett. 2018, 75, 105-111. [CrossRef]

11. Gonzalez-Ovejero, D.; Martini, E.; Loiseaux, B.; Tripon-Canseliet, C.; Mencagli, M.; Chazelas, J.; Maci, S. Basic Properties of Checkerboard Metasurfaces. IEEE Antennas Wirel. Propag. Lett. 2015, 14, 406-409. [CrossRef]

12. Vardaxoglou, Y. Metamaterial Structures for Antenna Applications; IET Semin. Metamater. Microw. (Sub) Millimetrewave Appl. 2008, 5-10. [CrossRef]

13. Nishimura, K.; Tsutsumi, M. Scattering of millimeter waves by metallic strip gratings on an optically plasma-induced semiconductor slab. IEEE Trans. Microw. Theory Tech. 1996, 44, 2231-2237. [CrossRef]

14. Lockyer, D.S.; Vardaxoglou, J.C. Transmission Through Optically Generated Inductive Grid Arrays. IEEE Trans. Microw. Theory Tech. 1999, 47, 1391-1397. [CrossRef]

15. Chaharmir, M.R.; Shaker, J.; Cuhaci, M.; Sebak, A. Novel Photonically-Controlled Reflectarray Antenna. IEEE Trans. Antennas Propagat. 2016, 54, 1134-1141. [CrossRef]

16. Nishimura, K. Electromagnetic wave scattering from an optically controlled infinite periodic rectangular cavity array with an electrically short period. In Proceedings of the IEEE International Symposium on Antennas and Propagation (APSURSI), Spokane, DC, USA, 3-8 July 2011; pp. 1871-1874. [CrossRef]

17. Tripon-Canseliet, C.; Chazelas, J. Nanoscale Microwave Engineering: Optical Control of Nanodevices; Wiley Blackwell: Hoboken, NJ, USA, 2014. [CrossRef]

18. Krraoui, H.; Mejri, F.; Aguili, T. Nondestructive measurement of complex permittivity by a microwave technique: Detection of contamination and food quality. J. Electromagn. Waves Appl. 2017, 31, 1638-1657. [CrossRef]

19. Fenner, R.A.; Rothwell, E.J.; Frasch, L.L. A Comprehensive Analysis of Free-Space and Guided-Wave Techniques for Extracting the Permeability and Permittivity of Materials Using Reflection-Only Measurements. Radio Sci. 2012, 47. [CrossRef]

20. Joseph, J.C. Multiple Angle of Incidence Measurement Technique for the Permittivity and Permeability of Lossy Materials at Millimeter Wavelengths. Master's Thesis, Air Force Inst. Technology, Wright-Patterson Air Force Base, OH, USA, 1986; pp. 29-36.

21. Sauerer, B. Optically CW-Induced Losses in Semiconductor Coplanar Waveguides. IEEE Trans. Microw. Theory Tech. 1989, 37, 139-149. [CrossRef]

(C) 2020 by the authors. Licensee MDPI, Basel, Switzerland. This article is an open access article distributed under the terms and conditions of the Creative Commons Attribution (CC BY) license (http://creativecommons.org/licenses/by/4.0/). 